\title{
KSHV targets multiple leukocyte lineages during long-term productive infection in NOD/SCID mice
}

\author{
Christopher H. Parsons, ${ }^{1}$ Laura A. Adang, ${ }^{1}$ Jon Overdevest, ${ }^{1}$ Christine M. O'Connor, ${ }^{1}$ \\ J. Robert Taylor Jr., ${ }^{1}$ David Camerini, ${ }^{2}$ and Dean H. Kedes ${ }^{1}$

\begin{abstract}
${ }^{1}$ Myles H. Thaler Center for AIDS and Human Retrovirus Research, Departments of Microbiology and Internal Medicine, University of Virginia Health Systems, Charlottesville, Virginia, USA. 2Department of Molecular Biology \& Biochemistry, Center for Immunology, and Center for Virus Research, School of Biological Sciences, University of California, Irvine, California, USA.
\end{abstract}

\begin{abstract}
To develop an animal model of Kaposi sarcoma-associated herpesvirus (KSHV) infection uniquely suited to evaluate longitudinal patterns of viral gene expression, cell tropism, and immune responses, we injected NOD/SCID mice intravenously with purified virus and measured latent and lytic viral transcripts in distal organs over the subsequent 4 months. We observed sequential escalation of first latent and then lytic KSHV gene expression coupled with electron micrographic evidence of virion production within the murine spleen. Using novel technology that integrates flow cytometry with immunofluorescence microscopy, we found that the virus establishes infection in murine B cells, macrophages, NK cells, and, to a lesser extent, dendritic cells. To investigate the potential for human KSHV-specific immune responses within this immunocompromised host, we implanted NOD/SCID mice with functional human hematopoietic tissue grafts (NOD/SCID-hu mice) and observed that a subset of animals produced human KSHV-specific antibodies. Furthermore, treatment of these chimeric mice with ganciclovir at the time of inoculation led to prolonged but reversible suppression of KSHV DNA and RNA levels, suggesting that KSHV can establish latent infection in vivo despite ongoing suppression of lytic replication.
\end{abstract}

\section{Introduction}

Over the past decade, studies have implicated human herpesviruses as the causative agents for a number of illnesses arising in patients with compromised immune systems related to HIV-1 infection or receipt of immunosuppressive therapies (1-3). A $\gamma$ herpesvirus, Kaposi sarcoma-associated herpesvirus (KSHV or HHV8), is the etiologic agent of KS, the most common AIDS-associated malignancy worldwide, as well as other malignant lymphoproliferative processes, including primary effusion lymphoma and multicentric Castleman disease (4-6). Although initiation of highly active antiretroviral therapy for HIV-1 infection has reduced the incidence of KSHV-related complications in this population (7), prevention or regression of tumors is often incomplete, and therapeutic alternatives, including systemic chemotherapy, or reductions in antirejection therapies are less effective $(8,9)$.

In vitro experiments and transgenic animal models have begun to elucidate the potential roles of individual KSHV proteins (2). However, a more thorough understanding of the relative contribution of these proteins to host-viral interactions and KSHV-related disease hinges on the creation of an in vivo model supporting whole-virus infection, latent and lytic viral gene expression, and a

Nonstandard abbreviations used: $B F$, brightfield; $C_{t}$, cycle threshold; GCV, ganciclovir; HPC, hematopoietic progenitor cell; IFA, immunofluorescence-based assay; KS, Kaposi sarcoma; KSHV, Kaposi sarcoma-associated herpesvirus; LANA, latency-associated nuclear antigen; MIFC, multispectral imaging flow cytometry; N/C, nuclear to cytoplasmic (ratio); NOD/SCID-hu, xenotransplanted NOD/SCID; ORF, open reading frame; qPCR, quantitative real-time PCR; qRT-PCR, quantitative reverse transcriptase real-time PCR; RTA, replication and transcription activator; TEM, transmission electron microscopy; UV-KSHV, UV-inactivated KSHV

Conflict of interest: The authors have declared that no conflict of interest exists. Citation for this article: J. Clin. Invest. 116:1963-1973 (2006). doi:10.1172/JCI27249. larger array of target cell and tissue types. Such an in vivo approach would also provide a more rigorous model for the development of effective anti-KSHV therapeutics. To date, attempts to establish long-term de novo infection within relevant target cells following the introduction of KSHV to either primate or chimeric hosts have been unsuccessful (10-12).

In this study, we injected NOD/SCID mice with purified KSHV and demonstrated latent and lytic viral gene expression within murine spleens and long-term infection within specific leukocyte cell populations. Parallel experiments employing NOD/SCID mice implanted with functional human hematopoietic tissue grafts (NOD/SCID-hu model) revealed a human KSHV-specific humoral immune response within a subset of infected animals, and administration of short-term "preemptive" antiviral therapy to these chimeric mice resulted in long-term but reversible suppression of viral DNA and mRNA levels.

\section{Results}

Longitudinal increase in latent and lytic viral transcripts within NOD/SCID mice following intravenous injection of KSHV. To ascertain whether KSHV establishes long-term infection within an immunocompromised murine host, we injected NOD/SCID mice intravenously with either KSHV or UV-inactivated KSHV (UV-KSHV) once weekly for 3 weeks and followed the levels of viral DNA and RNA longitudinally (below). Amplification of DNA sequences within the KSHV open reading frame 73 (ORF73) encoding the latency-associated nuclear antigen (LANA) (13) from total DNA extracted from NOD/ SCID spleen tissue 24 hours after injection revealed approximately equivalent amounts of KSHV DNA within animals injected with UV-KSHV or KSHV (Figure 1A). At all subsequent time points, however, only the mice injected with untreated virus showed steadi- 
B



A

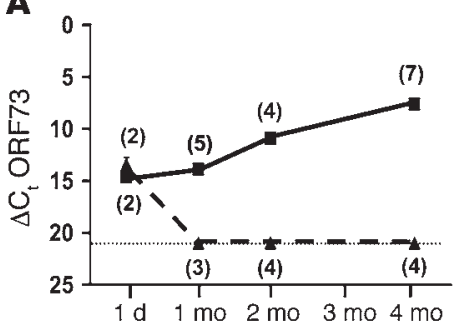

C

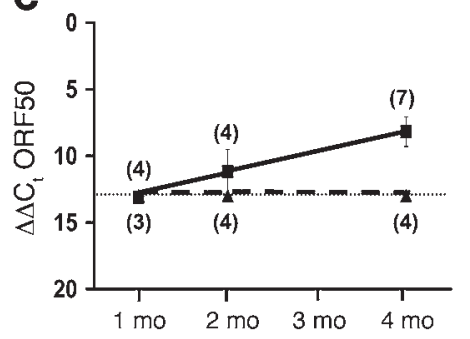

D

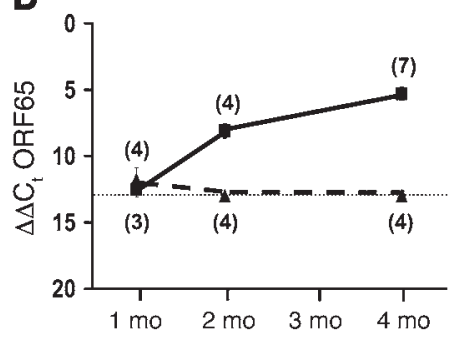

Figure 1

Sequential increases in KSHV genomic DNA, latent (ORF73) and lytic (ORF50 and ORF65) transcripts within the spleens of KSHV-injected NOD/SCID mice. Mice were administered 3 weekly doses of KSHV (squares, solid lines) or UV-KSHV (triangles, dashed lines) intravenously and euthanized 1 day (DNA only) and 1, 2, and 4 months following the third injection. (A) Genomic KSHV DNA values were determined using qPCR to calculate the $\Delta \mathrm{C}_{t}$, representing KSHV $\mathrm{C}_{t}$ normalized to mouse GAPDH $\mathrm{C}_{t}$ (mean of triplicate determinations for each) for each sample. (B-D) Total splenic RNA was subjected to qRT-PCR using primers specific for ORFs 73 (B), 50 (C), and 65 (D) as well as mouse GAPDH. $\Delta \Delta C_{t}=\left(\Delta C_{t(\mathrm{G})}\right)-\left(\Delta C_{t(\mathrm{~K})}\right)$, where $\Delta C_{t(\mathrm{~K})}$ and $\Delta C_{t(\mathrm{G})}$ are the differences between the $\mathrm{C}_{t}$ of each sample (mean of duplicate determinations) without and with reverse transcriptase (RT) for KSHV (K) and GAPDH $(\mathrm{G})$, respectively. Each symbol represents the mean and SE of $\Delta \mathrm{C}_{\mathrm{t}}(\mathrm{A})$ or $\Delta \Delta C_{t}(B-D)$. Numbers of mice are indicated in parentheses. Graph-wide dotted lines mark the lower limit of sensitivity of each assay. Of note, UV-KSHV did not establish infection within either primary dermal microvascular endothelial cells (pDMVECs) or HeLa cells in vitro (not shown).

ly increasing levels of KSHV DNA, reaching a viral genome copy to cell ratio of approximately $1: 300$ by 4 months after infection. In contrast, the levels of viral DNA from mice injected with UV-KSHV fell below the threshold of detection after the 1-day time point, likely reflecting degradation of the inactivated input virus.

To assess viral transcription programs within the NOD/SCID mice, we used quantitative reverse transcriptase real-time PCR (qRT-PCR) to amplify latent and lytic KSHV transcripts from total splenic RNA. Elevated levels of the ORF73 transcript were present beginning as early as 1 month after injection with KSHV and increased by approximately 32 -fold over the subsequent 3 months (Figure 1B). Both immediate early lytic (ORF50, encoding the lytic switch protein RTA [replication and transcription activator]; ref. 14) and late lytic (ORF65, encoding the structural protein SCIP [small capsomerinteracting protein]; ref. 15-17) genes displayed a delayed pattern of transcription, reaching detectable levels 2 months after injection and increasing at later time points (Figure 1, C and D). In contrast, the levels of all 3 transcripts in the UV-KSHV-infected mice remained at or below the level of sensitivity of the assays (Figure 1, B-D).

Expression of KSHV proteins within NOD/SCID cells. To observe the pattern of KSHV protein expression within NOD/SCID spleens, we evaluated cells for the expression of $2 \mathrm{KSHV}$ proteins: LANA and an envelope glycoprotein encoded during late lytic replication, K8.1, using immunofluorescence-based assays (IFAs). We found that $0.5-1 \%$ of host spleen cells from mice 4 months after KSHV injection exhibited a punctate intranuclear staining pattern (dots) consistent with LANA. In contrast, we saw no staining over background in spleens from either KSHV-injected mice at early time points or UV-KSHV-injected mice at any time point (Figure 2, A-D). The majority of LANA-positive cells within most organs contained only a small number of LANA dots (Figure 2), an observation consistent with published data suggesting that the majority of latently infected cells within infected human tissue contain few copies of the viral episome (18). In contrast, only rare (0.01-0.1\%) cells within parallel sections from KSHV-treated mice exhibited peripheral staining consistent with membrane or cytoplasmic staining of K8.1 in cells supporting lytic viral replication (19). Sections from untreated animals or mice injected with UV-KSHV showed low background levels of reactivity $(<0.001 \%$ of cells) after incubation with the same antibody (Figure 2, C and D).

Examination of 50 spleens representing 4 different experiments with NOD/SCID (25 animals) and NOD/SCID-hu mice (25 animals) revealed that $1 \mathrm{NOD} / \mathrm{SCID}$ and $2 \mathrm{NOD} / \mathrm{SCID}$-hu organs were grossly enlarged (approximately 10-fold increase in their largest dimension) relative to control organs (not shown). Moreover, these organs contained multiple clusters of LANA-positive cells, many of which contained 3-10 discrete intranuclear LANA dots, as well as an overall proportion of LANA-positive cells (20-30\%) that was significantly higher than in infected mice with normal-sized spleens. Although these events may represent KSHV-induced cell transformation or an expansion of infected cells within these organs, the rarity of these splenic tumors prevented more thorough analyses.

Virion production within NOD/SCID spleens following KSHV injection. Expression of late lytic structural genes (Figures 1 and 2) suggested ongoing virion assembly and production within host cells. Exploring this issue with transmission electron microscopy (TEM), we detected viral capsids and mature virions within a small number of splenocytes from KSHV-injected mice (Figure 3, A-C) but not UV-KSHV-injected (or uninfected) mice (not shown). Similarly, spleens from KSHV-injected mice implanted with human tissue 

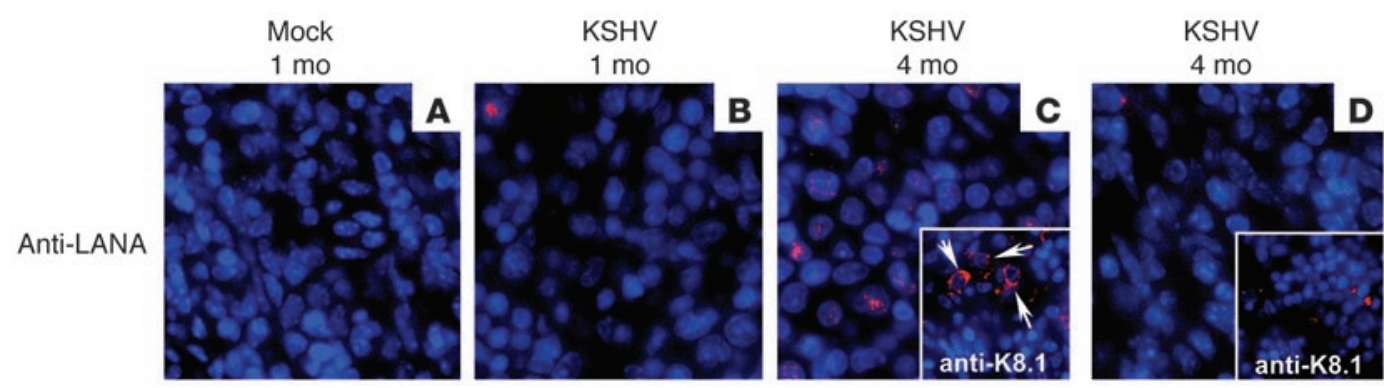

\section{Figure 2}

KSHV latent and lytic protein expression escalated over time following intravenous injection of NOD/SCID mice. NOD/SCID spleens were collected 1 month (A and B) and 4 months (C and D) after intravenous injection with PBS (A), KSHV (B and C), and UV-KSHV (D). Spleen sections were incubated with DAPI (blue) and mAbs to either LANA (A-D, large panels) or K8.1 (C and $\mathbf{D}$, insets) and the images merged to assess colocalization. Solely to emphasize the qualitative aspects of the LANA staining pattern, we chose $\mathbf{C}$ from a mouse spleen with one of the highest overall infection rates (3\%). Staining characteristic of cytoplasmic or cell-surface localization of K8.1 was also evident within rare cells (approximately $0.01 \%$ ) in sections from KSHV-injected mice (C, inset, arrows) while no staining over background staining was observed within sections from UV-KSHV-injected mice (D, inset). Original magnification, $\times 40$ (large panels); $\times 20$ (insets).

(below for details) contained viral particles (Figure 3, D-F), and additional magnification (Figure 3, D-F) distinguished ultrastructural features within these particles characteristic of herpesviruses, including condensed DNA, angular icosahedral capsids, a bilayered lipid envelope surrounding a tegument layer, and finger-like projections representing surface glycoproteins.

Identifying the cellular targets of KSHV infection in the spleens of NOD/ $S C I D$ mice. Studies of KSHV in human patients and murine homologs in immunocompetent mice have revealed that specific leukocyte subsets within lymphoid organs support KSHV latency, including B cells, monocyte lineage cells, and dendritic cells (20-23). Using flow cytometry, we looked for these leukocyte cell populations within the NOD/SCID spleens and found discernible populations potentially relevant to KSHV pathogenesis, including $\mathrm{B}$ cells (B220 CD11b-Ly49-CD11 $\mathrm{c}^{-}$), macrophages (CD11 ${ }^{+} \mathrm{CD} 11 \mathrm{c}^{+/-}$


ure 4). Earlier work has also demonstrated the presence of NK cells in NOD/SCID mice (24) and KSHV-specific NK cell responses in human patients (25). We also detected a small (approximately 5-7\% of total) but well-defined population of cells expressing markers consistent with NK cells (Figure 4; Ly49 ${ }^{+} \mathrm{IL}-2 \mathrm{R} \beta^{+} \mathrm{CD} 11 \mathrm{~b}^{-} \mathrm{CD} 11 \mathrm{c}^{-}$ B220- $\left.{ }^{-} 3^{-}\right)$. As expected for NOD/SCID spleens (26), the $\mathrm{CD}^{+} \mathrm{T}$ cell population was negligible (Figure $4 \mathrm{H}$ ).

Since our data indicated that the KSHV infection rate was relatively low in NOD/SCID mouse splenocytes (0.5-1\%), accurately identifying subpopulations containing the virus necessitated the development of an assay with sufficient sensitivity and specificity for this task. We employed a novel image-based flow cytometric approach

\section{Figure 3}

Thin section EM of NOD/SCID-hu spleens demonstrated virion production 3 months after infection with KSHV. (A) Angular C capsid (arrow) within cytoplasmic vesicle. (B) A capsids. (C) Cytoplasmic virion. (D) Cytoplasmic virion likely sectioned asymmetrically, allowing good visualization of angular capsid. (E) Virion within cytoplasmic vesicle likely during egress toward plasma membrane $(\mathrm{pm})$. (F) Released virion in extracellular space(es). (G-I) Identical to images D-F, respectively, but with an additional $\times 2.5$ magnification to reveal subviral structural components. t, tegument; c, capsid; gp, glycoproteins; e, envelope; v, vesicle; $d$, viral DNA. Original magnification, $\times 28,000$. Scale bars, $0.2 \mu \mathrm{m}$. (multispectral imaging flow cytometry [MIFC]) that has greater specificity and sensitivity than standard methods. This technology allows high-throughput quantitative and qualitative characterization of single cells within a population by assessing a combination of morphology and immunofluorescence staining patterns (see Methods and Supplemental Methods; supplemental material available online with this article; doi:10.1172/JCI27249DS1). In this way, we identified populations of cells displaying the intranuclear punctate staining pattern characteristic of LANA simultaneously with specific cell-surface markers. We found that approximately $0.9 \%$ of all splenocytes collected from NOD/SCID mice 3 to 4 months after injection with KSHV revealed punctate intranuclear LANA staining (Figure 5, A and C) while spleen cells from UV-KSHVinjected mice revealed no specific staining pattern (Figure 5B). LANA positivity was demonstrated for $\mathrm{B} 22 \mathrm{O}^{+}, \mathrm{Ly}_{4} 9^{+}, \mathrm{CD}_{11 \mathrm{~b}}+$, and


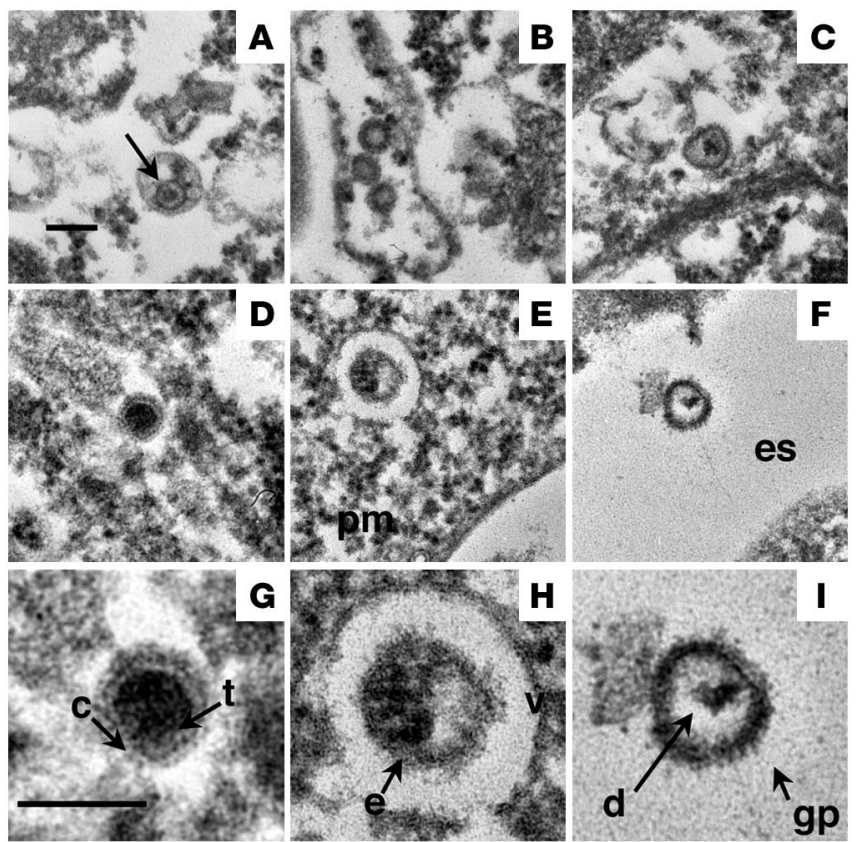
A
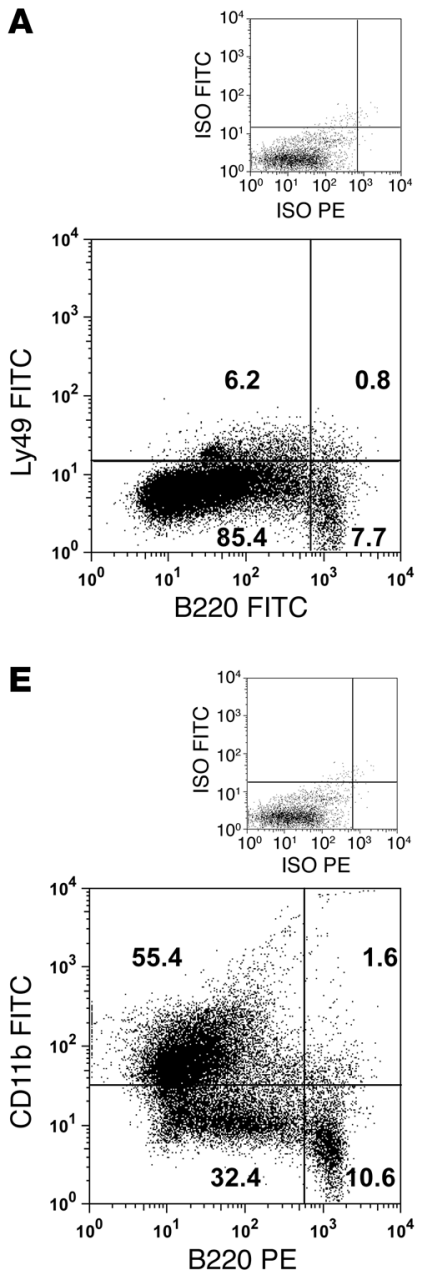

B
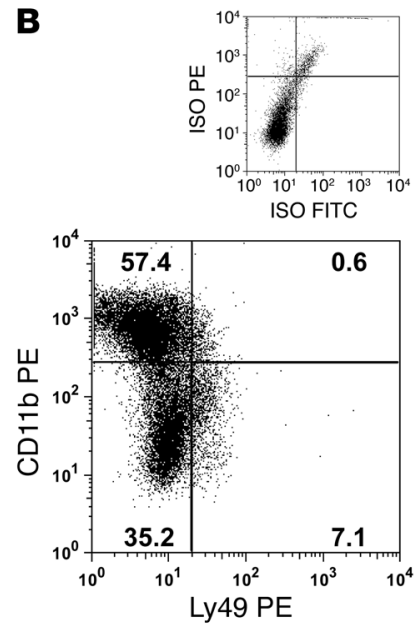

$\mathbf{F}$
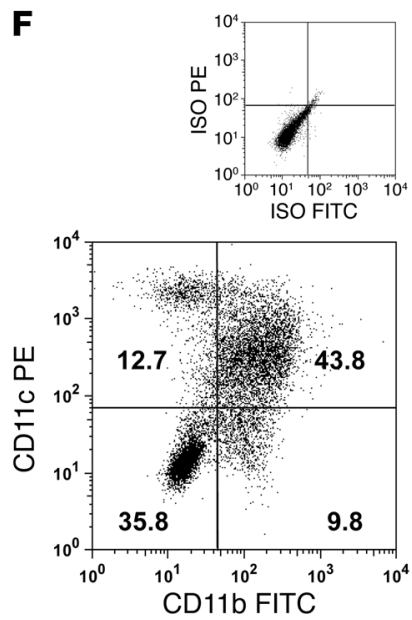

C

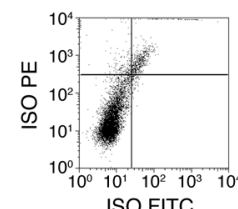

D
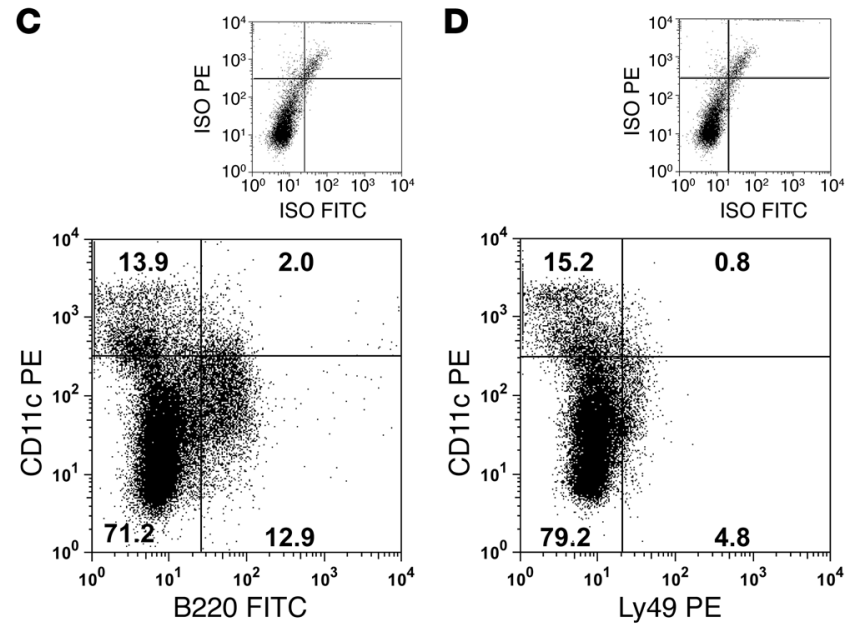

G

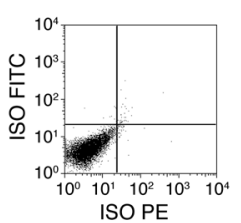

H
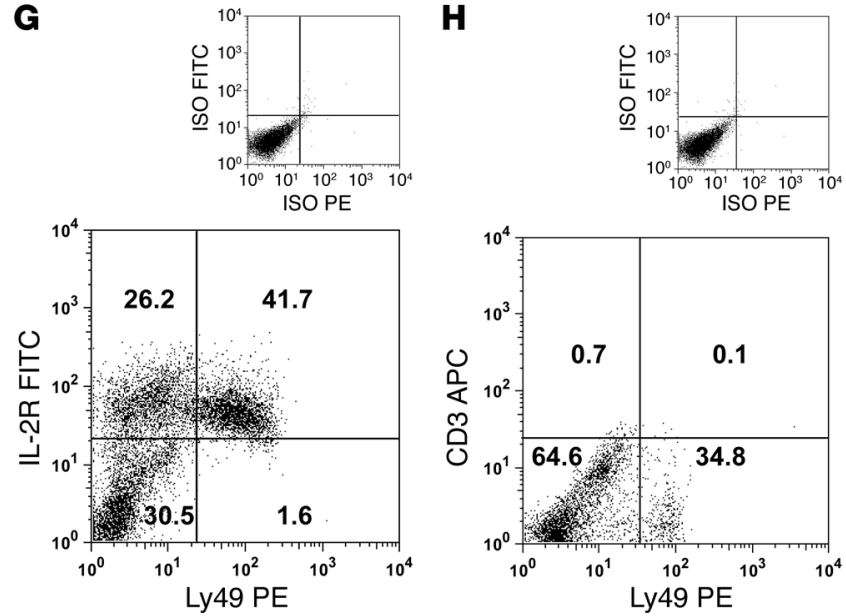

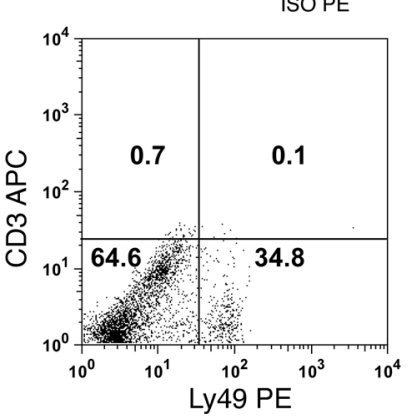

Figure 4

Identification of murine leukocyte subpopulations within NOD/SCID spleens. Splenocyte suspensions from KSHV-infected NOD/SCID mice were incubated with antigen-specific monoclonal antibodies (large panels) or their respective isotype controls (small panels). (A-F) Approximately $10,000$ total live cells were evaluated for the coexpression of B220, Ly49, CD11b, and CD11c for all 2-way combinations. (G and $\mathbf{H})$ In separate experiments gating only on the lymphocyte population, coexpression was observed for Ly49 and IL-2R $\beta$ (G), but not Ly49 and CD3 (H).

and D). Since $\mathrm{CD} 34^{+}$human hematopoietic progenitor cells (HPCs) support latent KSHV infection (27), we looked for $\mathrm{LANA}^{+}$cells within a population expressing CD117 (c-kit), an antigen expressed at high levels on murine HPCs (28), but found that these cells did not express LANA. Nearly all of the LANA ${ }^{+}$cells expressed one of the surface markers we examined in these experiments (Figure 5D). Furthermore, the majority of positive cells exhibited only a limited number of LANA dots (Figure 5A), consistent with our findings in the splenic tissue sections (Figure 2C).

Combining our flow cytometry data with additional morphologic parameters, including area (Figure 5E) and nuclear to cytoplasmic $(\mathrm{N} / \mathrm{C})$ ratio (Figure $5 \mathrm{~F}$ ), we verified the identity of 4 distinct (nonoverlapping) populations of $\mathrm{LANA}^{+}$cells within NOD/SCID spleens: B220 ${ }^{+}$staining identified small cells with high N/C ratios (B cells); Ly49+ beaded surface staining identified intermediately sized cells with low N/C ratios (NK cells); CD11b+ staining identified intermediately sized cells with low $\mathrm{N} / \mathrm{C}$ ratios (macrophages); and $\mathrm{CD} 11 \mathrm{c}^{+}$staining identified intermediately sized cells with high $\mathrm{N} / \mathrm{C}$ ratios (dendritic cells). LANA-negative populations included small cells exhibiting $\mathrm{CD}^{+}$surface staining with high $\mathrm{N} / \mathrm{C}$ ratios ( $\mathrm{T}$ cells) and large cells with $\mathrm{CD} 117^{+}$surface staining with high $\mathrm{N} / \mathrm{C}$ ratios (HPCs). In summary, KSHV targeted B cells, NK cells, macrophages, and dendritic cells but not murine T cells or HPCs in infected NOD/SCID mice (Table 1). Since MIFC demonstrated that these 4 populations were distinct and they combined to approximately $100 \%$ of the LANA-positive cells, we concluded that together they accounted for nearly all of the latently infected cells within the NOD/SCID spleens (Figure 5D).

Generating KSHV-specific human IgG responses in a NOD/SCID-bu model following KSHV infection. To determine whether a xenotransplanted variant of the animal model would support KSHV-specific human antibody responses following infection, we implanted NOD/SCID mice with human fetal bone, thymus, and skin (29). We then measured total human IgG in serum samples from implanted mice and unimplanted controls at monthly intervals to assess their capacity to generate human IgG. Two months after implantation, levels of human IgG in the NOD/SCID-hu mice were at least 700- to 1,000-fold greater than the nonspecific back- 
A



C

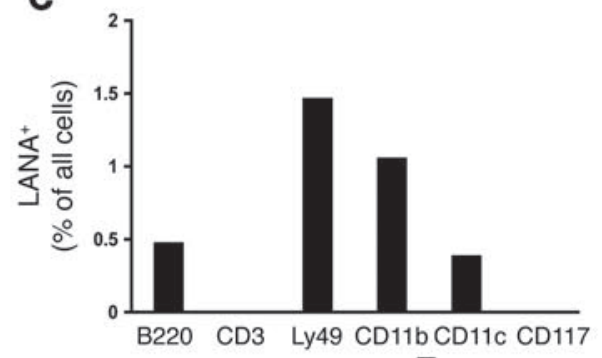

E

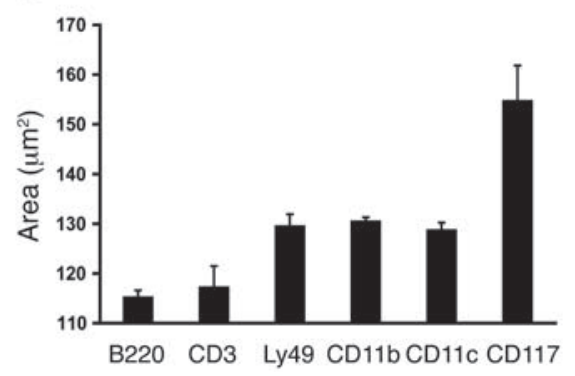

KSHV
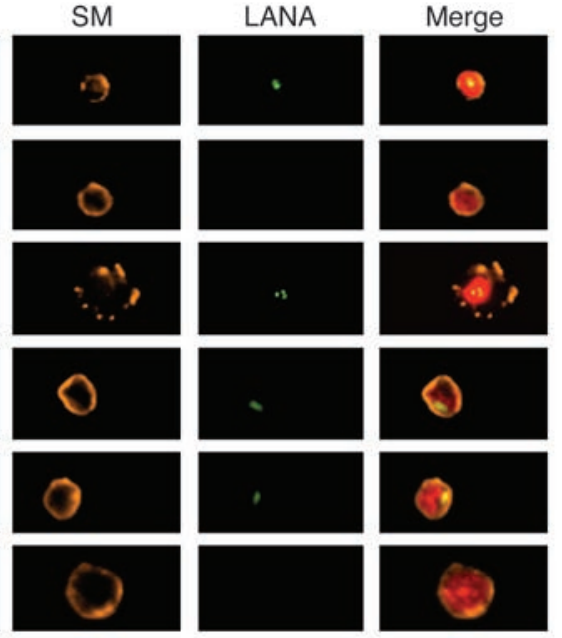

$\mathbf{F}$
B
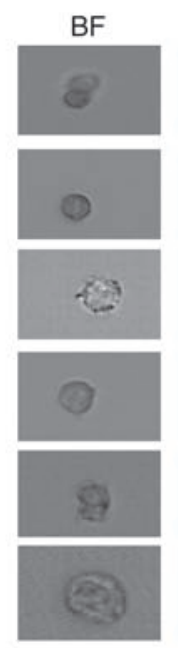

D

G

UV-KSHV
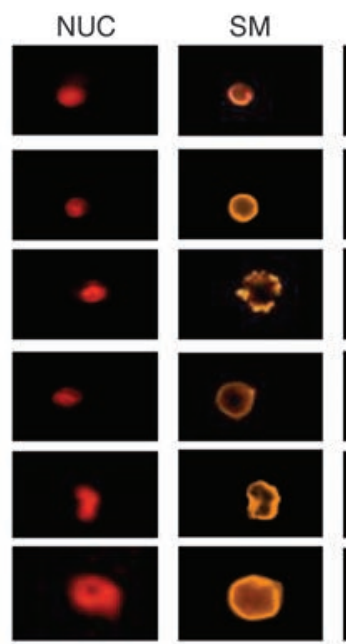
$$
\text { . }
$$

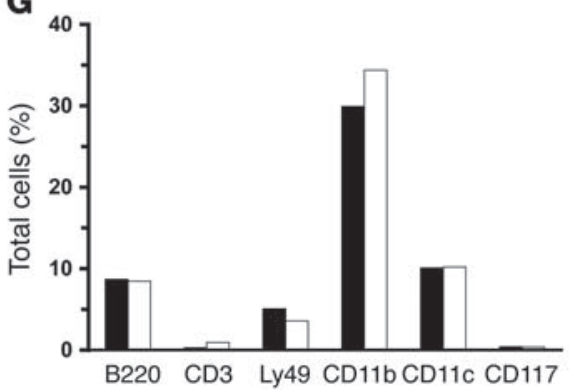

\section{Figure 5}

Distinct NOD/SCID spleen cell populations were latently infected with KSHV. (A) For cells from mice injected with KSHV, intranuclear LANA staining was noted in the $\mathrm{B}_{220^{+}}, \mathrm{Ly} 49^{+}, \mathrm{CD} 11 \mathrm{~b}^{+}$, and $\mathrm{CD} 11 \mathrm{C}^{+}$but not $\mathrm{CD} 3^{+}$or CD117+ populations. (B) Analogous populations of cells from mice receiving UV-KSHV revealed no specific staining suggestive of LANA. (C) The proportion of LANA+ cells within the positive populations in $\mathbf{A}$. (D) Four populations (see also text and Table 1 for details) accounted for nearly all LANA ${ }^{+}$cells. (E and $\mathbf{F}$ ) BF imaging and specific nuclear staining with DRAQ5 (NUC) allowed determinations of area (E) and N/C ratios (F) for individual cells. $P<0.001$ for mean size comparisons between small-sized $\left(\sim 115 \mu \mathrm{m}^{2} ; \mathrm{CD}^{+}\right.$and B220+) and intermediate-sized $\left(\sim 130 \mu \mathrm{m}^{2}\right.$; Ly49+, CD11 $\mathrm{b}^{+}$, and CD11C $\left.{ }^{+}\right)$cells and between intermediate-sized and large-sized $\left(\sim 155 \mu \mathrm{m}^{2}\right.$; CD117+) cells. $P<0.001$ for N/C comparisons between populations with low $\left(\sim 0.31\right.$; Ly49+ and CD11 $\left.\mathrm{b}^{+}\right)$and high


ent cell types are similar in mice 4 months after injection with either KSHV (white bars) or UV-KSHV (black bars). SM, surface marker.

ground levels in unimplanted controls (Figure 6A). Using a LANA IFA (30), we assayed serum samples from 13 chimeric animals (representing 2 xenotransplantation experiments) 3 months after their infection with KSHV for the presence of human anti-LANA antibodies and found that 3 of these samples yielded punctate intranuclear staining of target cells that was indistinguishable from the pattern generated with either serum from patients with KS or polyclonal anti-LANA antibodies (Figure 6, B, D, and E, respectively). The mice sera were nonreactive with KSHV-negative BJAB cells (not shown). Application of anti-mouse (rather than anti-human) secondary antibodies yielded no reactivity (Figure 6C), arguing against the presence of murine anti-LANA antibodies that may have arisen from reversion of the SCID phenotype (26). Remarkably, the sera with the highest titers of human anti-LANA antibodies (1:80) came from the 2 animals exhibiting the largest fraction of LANA-reactive cells within their spleens (not shown).

Effect of systemic ganciclovir administration on KSHV gene expression. To test the effects of an antiviral compound on the establishment and maintenance of de novo infection within NOD/SCID-hu mice, we administered the viral replication inhibitor ganciclovir 
Table 1

Identification of 6 unique NOD/SCID splenic cell types by MIFC

\begin{tabular}{|c|c|c|c|c|}
\hline $\begin{array}{l}\text { Surface } \\
\text { marker }\end{array}$ & Size & $\mathrm{N} / \mathrm{C}$ ratio & $\begin{array}{l}\text { Surface } \\
\text { staining }\end{array}$ & Identity \\
\hline CD3 & Small & Large & Continuous & $\mathrm{T}$ cell \\
\hline B220 & Small & Large & Continuous & B cell \\
\hline Ly49 & Intermediate & Small & Beaded & NK cell \\
\hline CD11b & Intermediate & Small & Continuous & Macrophage \\
\hline CD11C & Intermediate & Large & Continuous & Dendritic cell \\
\hline CD117 & Large & Large & Continuous & Stem cell \\
\hline
\end{tabular}

(GCV) intraperitoneally to a cohort of mice before and during the period of KSHV injection (Methods). DNA levels for GCV-treated mice were significantly lower than for untreated animals throughout the time course but greater than for UV-KSHV-injected mice at the later time points (Figure 6A). GCV-treated mice exhibited delayed ORF73 mRNA expression, detectable in our assay only at the 3-month time point (Figure 6B). Suppression of lytic (ORF50 and ORF65) transcript levels by the transient GCV treatment was even more pronounced, with no discernable difference between UV-KSHV-injected and GCV-treated mice even 4 months after drug withdrawal (Figure 6, C and D).

\section{Discussion}

These data are the first, to our knowledge, to demonstrate that murine hosts can support long-term de novo KSHV infection, including the establishment of latency, progressive expansion of viral load, and the assembly of virions. Our results also indicate that KSHV infects specific murine leukocyte populations resembling those targeted by the virus in humans. Furthermore, infection of NOD/SCID mice implanted with human hematopoietic tissue grafts evokes a human KSHV-specific antibody response in a subset of animals that recapitulates the most common serologic reactivity present in infected patients. Finally, treatment of these chimeric mice with preemptive GCV reversibly suppresses viral genome expansion and gene expression over a 3-to 4-month time course, suggesting that the initial establishment of latency in the model is independent of productive lytic replication.

Although existing models of nonhuman primate or murine $\gamma$ herpesviruses are helpful in understanding the parallels between these viruses and KSHV, their ultimate utility remains limited by intrinsic differences in their lytic reactivation dynamics, immune responses, and pathologic correlates (31-35). Similarly, animal model systems for KSHV have, thus far, focused on overexpression of single viral oncogenes in transgenic systems (36-38) or the in vivo growth of malignant cell lines, for which it is difficult to evaluate de novo viral tropism and gene expression (11, 19, 39-41). Although these latter models are critical for providing insights into various aspects of KSHV pathogenesis, a more complete understanding of infection, cellular tropism, and the interplay of viral gene expression in the host depends on a model that supports stable infection with intact virus. To date, however, attempts to detect lytic KSHV replication, immune responses, and related pathology within an immunocompromised large animal model system have proven unsuccessful (12). Three previous small animal studies have described KSHV gene expression within human cells, including a xenotransplantation model optimized for the study of HIV-1 infection (10), another that supported propagation of human PBMC (11), and a third that employed direct viral infection of implanted human skin (42). However, these models are unable to address more complex issues related to cell tropism and long-term KSHV gene expression due to limitations of the host cell repertoire $(10,11,42)$, duration of viral replication with declining viral DNA titers over time (10), or a lack of discernable de novo infection (11, 42). Our results also contradict published data suggesting that the tethering of KSHV episomes to human host cell chromatin, but not to murine chromatin, relates to the binding of LANA to regions of the chromosome-associated proteins DEK and methyl CPG-binding protein MECP2 that are not well conserved in their respective murine homologs (43). As the authors suggest, however, levels of these proteins may not be sufficiently high to show LANA interaction in their experiments. Alternatively, LANA may interact with other murine chromosome-associated proteins or proteins expressed in a limited repertoire of cells within the mouse.

Our PCR analysis of NOD/SCID spleens following intravenous injection of KSHV suggests that latency is established within 1 month of injection (Figure 1B). The initial detection of the latencyassociated ORF73 transcripts was followed by a sustained rise in the lytic cycle ORF50 and ORF65 transcripts (Figure 1, C and D). Of note, the rate of rise and absolute levels of ORF65 mRNA were appreciably greater than for ORF50. Since ORF65 encodes SCIP, a protein present in approximately 900 copies per virion (16), this difference may reflect the virus' need for higher mRNA levels for this late lytic structural gene than for ORF50, which encodes the immediate early transcription factor, RTA. In both cases, the rise

\section{Figure 6}

KSHV-specific human antibody production in NOD/ SCID-hu mice inoculated with KSHV. (A) Compared with unimplanted mice $(U)$, sera from NOD/ SCID mice receiving human tissue implants (I) contained detectable amounts of total human IgG. (B-E). Serum (1:80) from an implanted and KSHVinjected mouse probed with either anti-human (B) or anti-mouse (C) secondary antibodies. Serum from a human KSHV-infected patient (1:640) (D) or rabbit polyclonal anti-LANA antiserum (1:40) (E) followed by anti-human or anti-rabbit secondary antibodies conjugated to Texas Red, respectively. Original magnification, $\times 60$.
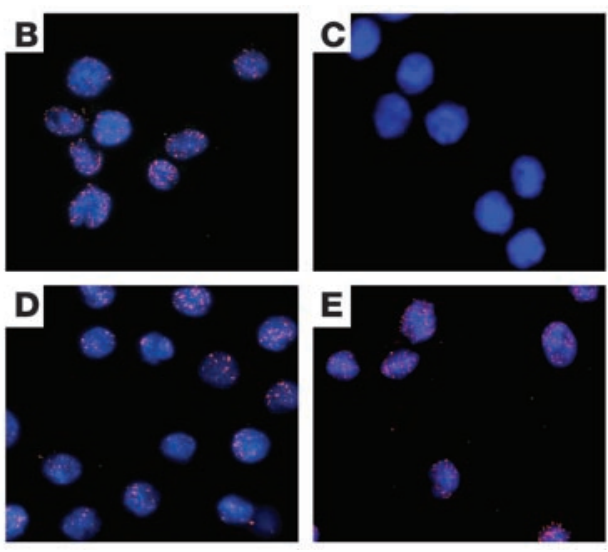
A


C

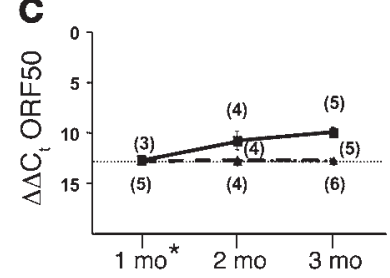



Figure 7

Increases in KSHV genomic DNA in NOD/SCID-hu mouse spleens were inhibited temporally and quantitatively by pretreatment with GCV. Mice received daily (days -24 to +1 ) intraperitoneal administration of either GCV (diamonds, dotted line) or PBS (squares, solid line) during the 3-week KSHV infection period. A third group of mice received UV-KSHV (triangles, dashed line). (A) Mean $\Delta \mathrm{C}_{\mathrm{t}}$ values of genomic KSHV DNA were calculated as in Figure 1. ${ }^{*}$ Data from 1-month GCV-treated mice were not collected, indicated by the discontinuous line. (B-D). Mean $\Delta \Delta C_{t}$ values representing KSHV RNA determinations for ORFs 73 (B), 50 (C), and 65 (D) were calculated as in Figure 1. Each symbol represents the mean and SE of $\Delta C_{t}(A)$ or $\Delta \Delta C_{t}(B-D)$. Numbers of mice and the lower limit of sensitivity of the assays are indicated as in Figure 1.

in lytic transcripts likely reflects robust lytic replication within a small number of permissive cells, as suggested by IFA and TEM data from the infected spleens. Together, these observations also closely mimic viral gene expression patterns within human KS lesions, where only $1-5 \%$ of infected cells support spontaneous lytic gene expression, even in advanced disease (44-46).

With the visualization of ultrastructural elements (Figure 3 ) that are indistinguishable from those that we and others have described previously for KSHV within human cell lines and splenic tissue (16, 47), we provide, for what we believe is the first time, direct evidence for KSHV assembly within an in vivo model following systemic infection with purified virus. Although different members of the herpesvirus family are indistinguishable from one another based exclusively on ultrastructural characteristics, our data are highly suggestive of KSHV assembly, based on our corroborating molecular and IFA data, the complete absence of similar structures within tissue collected from UV-KSHV-injected control NOD/SCID mice or from KSHVinjected mice at earlier time points (not shown), and the seronegativity of our animals for a large number of other viruses (Methods).

Previous studies have applied quantitative real-time PCR (qPCR) assays to specific cell populations following flow cytometric sorting to define cell tropism for $\operatorname{KSHV}(10,48)$ and murine $\gamma$ herpesvirus 68 (MHV-68) (21) in vivo. Although potentially effective for model systems supporting high levels of viral replication and larger numbers of infected cells, PCR-based methods for determining cellular tropism become less reliable with lower levels of infection. Thus, detection of KSHV DNA by qPCR from even a small number of "contaminating" cells would be sufficient to incorrectly identify the main population as KSHV infected. Additionally, standard flow cytometry lacks the ability to confirm specific patterns of staining for individual cells and is, therefore, less able to exclude cells with aberrant but equally intense overall staining (L.A. Adang and D.H. Kedes, unpublished observations).

To more precisely distinguish NOD/ SCID splenocyte subpopulations and determine the specific cellular targets of KSHV infection, we employed MIFC, a novel approach (see Methods) that integrates multispectral imaging of individual cells with the statistical power of flow cytometry. Using morphologic parameters (Figure 4, E and F), we were able to characterize 6 distinct populations of murine spleen cells (Figure 4H): $\mathrm{B}$ cells, T cells, NK cells, macrophages, dendritic cells, and HPCs. Furthermore, we observed no significant differences in the cell-determinant staining patterns (Figure 4, A and B), morphologic characteristics (Figure 4, A, B, E, and F), or relative numbers of these populations (Figure 4G) between UV-KSHV- and KSHV-injected mice.

We next determined which of these 6 cell types demonstrated clear evidence of LANA expression (Figure 5, A and $B$, and Methods). Similar to reports for KSHV in human patients and MHV-68 in murine hosts $(20,22,23)$, B cell, macrophage and, to a lesser extent, dendritic cell populations contained $\mathrm{LANA}^{+}$cells. The aberrations in $\mathrm{T}$ cell receptor rearrangement characterizing the SCID phenotype contribute to an arrest of $\mathrm{B}$ and $\mathrm{T}$ cell maturation at early stages in NOD/SCID mice (26), and thus, the $\mathrm{LANA}^{+} \mathrm{B} 220^{+}$cells that we identified most likely represent the overriding pro-B cell subtype present within NOD/SCID spleens (26). Our experiments did not address whether cells at different stages of differentiation or activation were preferentially infected.

Notably, we also observed LANA ${ }^{+}$cells within the NK cell population, representing, to our knowledge, the first evidence for in vivo infection of NK cells by a $\gamma$ herpesvirus. Although others have recognized NK cells as potentially important for the control of KSHV replication (25), one report suggested that circulating NK cells were not an important reservoir for KSHV within human patients (49). The investigators in this earlier study did not, however, assess NK cells derived from noncirculating leukocyte compartments. Nevertheless, whether NK cells ultimately represent an important latent reservoir for KSHV or simply "innocent bystanders" within the NOD/SCID host remains unknown.

Although our findings suggest that KSHV infection of specific cell populations reflects preferential targeting by the virus, these findings could theoretically represent infection of locally permissive populations resulting from viral recognition of promiscuous ligands, such as heparin sulfate or $\alpha 3 \beta 1$ integrin $(50,51)$. However, relative infection rates among the different subpopulations did not parallel their relative abundance within the NOD/SCID spleens, and 2 distinct populations, $\mathrm{CD}^{+} \mathrm{T}$ cells and $\mathrm{CD} 117^{+} \mathrm{HPCs}$, revealed no $\mathrm{LANA}^{+}$staining (Figure 5A). Although these 2 latter cell types represented only small fractions of the total population of spleen cells, we were able to evaluate a sufficient number of each to rule out all but very rare incidences of infection. Furthermore, although we cannot absolutely exclude the inclusion of a small number of double- or multi-marker positive cells among the LANA ${ }^{+}$ 
populations, the MIFC data argue for the mutual exclusivity of each subpopulation of cell types by a number of criteria, including cell-surface marker profile (Figure 4) and the unique morphologic characteristics of each cell type (Figure 5 and Table 1).

Previous work has shown that SCID mice implanted with human hematopoietic tissue grafts (the SCID-hu model) harbor functional B cells capable of IgG-specific responses to antigen challenge (52). Our results indicate that xenotransplanted NOD/SCID mice injected with KSHV exhibit human IgG levels (Figure 6) commensurate with previously published data (52) and levels of viral mRNA and virion assembly that were indistinguishable from those in infected unimplanted NOD/SCID mice over the same time frame (Figures 1, 3, and 7). Of the infected NOD/SCID-hu mice, a subset exhibited human anti-LANA reactivity in a standard LANA IFA (Figure 6). Of note, these reactive sera came from mice with relatively large (3-5\% of total cells stained) populations exhibiting LANA expression by IFA within the splenic tissue (not shown). These results parallel data from human patients in whom circulating high-titer anti-LANA antibodies predict higher levels of viral replication (53).

A delay, but not elimination, of latent gene expression coupled with marked attenuation of lytic gene expression following preemptive GCV therapy in KSHV-injected NOD/SCID-hu hosts also supports the presence of de novo viral replication in the model and complements other published data showing the capability of this agent to inhibit lytic KSHV replication $(10,54)$. Our inability to detect viral protein expression or virion assembly within sections from GCV-treated animals (not shown) supports the idea that low-level lytic viral replication is necessary for horizontal viral spread (55). However, the observed increase in DNA and latent transcripts at later time points in these mice suggests that suppression of lytic replication at the time of viral inoculation fails to block the establishment and maintenance of latency. Given corroborating retrospective clinical data showing a reduction in the incidence of KS for patients receiving antiviral agents during periods of immune suppression (56), the NOD/SCID mouse model may lend itself to more detailed characterization of viral replication and horizontal spread in the face of chemoprophylactic regimens designed to prevent the clinical manifestations of KSHV infections in high-risk (seropositive) patients.

The present study focused on establishing an in vivo system that supports long-term KSHV infection and recapitulation of both latent and lytic gene expression rather than on examining viral pathology. Nevertheless, a small minority (3 of 50) of KSHV-injected mice, but none of the UV-KSHV-injected mice, developed gross splenic enlargement at late (3- to 4-month) time points. Although it appeared that these enlarged organs contained a significantly larger relative proportion of $\mathrm{LANA}^{+}$cells by IFA, ascertaining whether these findings reflect potential viral-induced tumorigenesis awaits additional long-term experiments.

Our results represent what we believe is the first successful effort to recapitulate long-term KSHV infection within an immunocompromised animal host. Infection in this model closely mimics the biology of the virus in patients, including latent infection of leukocyte populations relevant to KSHV pathogenesis and low levels of spontaneous lytic reactivation that results in virion production. Furthermore, the engraftment of human hematopoietic tissue into NOD/SCID mice prior to KSHV infection allows a KSHV-specific immune response, and infection of these chimeric mice is attenuated following treatment with systemic antiviral therapy. In addition to providing insights into cellular tropism and efficacy of antiviral agents, the NOD/SCID mouse model should aid future investigations of viral gene expression and viral spread within a dynamic in vivo system.

\section{Methods}

Preparation of KSHV. KSHV-positive cells (BCBL-1) were grown as described previously (57). To induce lytic reactivation of KSHV, cells were incubated with $0.6 \mathrm{mM}$ valproic acid (Sigma-Aldrich) for 5 days. Cell-free KSHV was collected as described previously (16) and passed through $0.45 \mu \mathrm{M}$ filters (Millipore) to remove any residual cells.

Titering of KSHV. Fresh aliquots from the KSHV preparations were used to determine the infectious titer of the virus on cultured HeLa cells. Briefly, 60,000 HeLa cells per well on fibronectin-coated chamber slides (BD Biosciences) were incubated with serial dilutions of the concentrated viral stock for 2 hours at $37^{\circ} \mathrm{C}$ in the presence of $8 \mu \mathrm{g} / \mathrm{ml}$ Polybrene (Sigma-Aldrich), washed, and then incubated in fresh media (DMEM; Invitrogen) supplemented with 10\% FBS. After 18-24 hours, cells were assayed for LANA reactivity with monoclonal rat anti-LANA antibodies (ABI) conjugated to fluorochrome ALEXA-488 according to the manufacturer's instructions (Invitrogen). See Supplemental Methods for additional details.

KSHV injections of NOD/SCID mice. Six- to eight-week-old NOD/SCID (NOD/LtSz-Prkdc scid/J or NOD/SCID; Jackson Laboratory) mice were purchased and maintained in pathogen-free conditions. All mice received $3 \mathrm{mg} / \mathrm{ml}$ trimethoprim-sulfamethoxazole (Sigma-Aldrich) in sterile water for routine consumption. Under general anesthesia, experimental mice received 3 weekly tail-vein injections, approximately 800,000 infectious particles per injection, from freshly resuspended viral stock. Control animals received viral aliquots from the same stock that were exposed to UV light as previously described (58).

Isolation of DNA and RNA from murine tissue. Tissue sections were stored immediately for future DNA or RNA analysis in liquid nitrogen or an RNase-free preservative (RNAlater; Ambion), respectively. DNA and RNA were isolated using QIAmp DNA Micro Kits (QIAGEN) and RNeasy Mini Kits (QIAGEN), respectively, as described by the manufacturer. Optical density readings were obtained for each DNA and RNA sample to guide equalization of sample sizes.

$q P C R$ and $q R T-P C R$. KSHV DNA from murine organs was measured by qPCR using standard methods to amplify a fragment within the latent KSHV gene ORF73. KSHV RNA was quantified using a standard qRT-PCR amplification protocol according to the manufacturer's instructions (Invitrogen and QIAGEN). cDNA-coding sequences for ORF73, ORF50, and ORF65 were amplified from $200 \mathrm{ng}$ of input cDNA incorporating concentrations of primers and probes as for DNA (see Supplemental Methods for primer sequences, details of GAPDH normalization for both DNA and RNA amplifications, and detailed protocols). Cycle threshold $\left(\mathrm{C}_{\mathrm{t}}\right)$ values were tabulated in triplicate for KSHV and GAPDH reactions and duplicated for all cDNA amplifications. Water controls verified minimal background. The sensitivity of our assay for KSHV amplification was determined using serial dilutions of an ORF73-containing plasmid within a stable (200 ng) amount of mouse DNA. Assuming each murine cell contains approximately 7 pg DNA, we were consistently able to detect 3 to 4 genome equivalents of KSHV within DNA extracted from $10^{5}$ cells.

Indirect immunofluorescence microscopy of murine tissue. NOD/SCID spleens were removed, fixed in $3 \%$ formalin for $48-72$ hours, embedded in paraffin, and sectioned for mounting on positively charged glass slides. Prior to staining, slides were deparaffinized in $100 \%$ xylene ( 3 times for 15 minutes each time), then sequentially rehydrated in serial dilutions 
of ethanol. For membrane permeabilization, slides were placed in Target Retrieval Solution (Dako) according to the manufacturer's instructions, incubated in a $95^{\circ} \mathrm{C}$ water bath for 30 minutes, and then allowed to cool to room temperature. After incubation in blocking reagent (PBS with $1 \% \mathrm{BSA}$ ) for 1 hour, sections were incubated for 2 hours with monoclonal rat anti-LANA or anti-K8.1 antibodies (ABI) diluted 1:20 in blocking reagent. After washes in PBS/0.05\% Tween-20 (Fisher Scientific), sections were incubated with 1:100 dilutions of secondary goat anti-rat IgG antibodies conjugated to Texas Red (Jackson ImmunoResearch Laboratories Inc.) and then with DAPI as above. Fluorescent images from serial planes, $1 \mu \mathrm{m}$ apart, for multiple representative fields on each slide were obtained after merging using the deconvolution function of Openlab software (version 3.1.5; Improvision).

TEM. To identify KSHV-associated viral particles within murine spleens, tissue from mice that were injected with either KSHV or UV-KSHV was processed for thin-section TEM as we have described previously (59) (see Supplemental Methods for additional details). Prior to these experiments, murine hosts were prescreened serologically for non-KSHV viral agents (see Supplemental Methods).

Flow cytometry. Spleen cell suspensions were created by filtering tissue through mesh screens (Sigma-Aldrich) into cold RPMI 1640. Red blood cells were lysed using a commercially available buffer according to the manufacturer's instructions (PharM Lyse; BD Biosciences - Pharmingen). All cells were washed 3 times in a blocking reagent containing $1 \times \mathrm{PBS}, 2 \% \mathrm{FBS}$, and $0.1 \%$ sodium azide, then incubated in $80 \mu \mathrm{l}$ of the blocking reagent with $0.5 \mu \mathrm{g} / 10^{6}$ cells of mouse Fc $\gamma \mathrm{III} / \mathrm{II}$ receptor blocker (BD Biosciences - Pharmingen) for 10 minutes. Cell aliquots were incubated for 45 minutes in the dark at $4{ }^{\circ} \mathrm{C}$ with fluorochrome-conjugated monoclonal antibodies, $0.5 \mu \mathrm{g}$ antibodies per $10^{6}$ cells, identifying murine HPC surface markers, including Ly49-FITC or PE (14B11), IL-2R $\beta$-PE (TM- $\beta 1$ ) (eBioscience), B220-FITC or PE (RA3-6B2), CD117PE (2B8), CD11b-FITC or PE (M1/70.15), CD11c-PE (N418), and CD3PE or APC (145-2C11) (CALTAG Laboratories), or equivalent concentrations of their respective isotype control antibodies. Stained cells were washed with PBS and resuspended in $2 \%$ paraformaldehyde prior to analysis using a FACSCalibur cytometer (BD Biosciences) and FlowJo (version 6.4; Tree Star) software.

MIFC. In 2 separate experiments conducted 3-4 months after the last injection of either KSHV or UV-KSHV, spleen cells were pooled from $6 \mathrm{KSHV}$ - and $5 \mathrm{UV}-\mathrm{KSHV}$-injected mice, respectively, from one experiment, and from $6 \mathrm{KSHV}$ - and $4 \mathrm{UV}-\mathrm{KSHV}$-injected mice from a second experiment. Isolated mononuclear cells were pooled and then incubated as above with B220-PE, CD117-PE, CD11b-PE, CD11c-PE, Ly49-PE, and CD3-PE to identify distinct hematopoietic cell populations (see text). Following a single wash in blocking reagent, cells were fixed in $500 \mu \mathrm{l}$ fixation buffer (eBioscience) for 20 minutes at room temperature. Cell pellets were incubated in permeabilization buffer containing $1 \times \mathrm{PBS}$, $0.1 \%$ saponin, and $0.1 \%$ sodium azide for 5 minutes at room temperature. After removal of the supernatant, the cell pellets were incubated for 45 minutes in the dark with monoclonal rat anti-LANA antibodies diluted 1:500 in permeabilization buffer. After washing, pellets were resuspended in PBS and incubated for 10 minutes at room temperature following the addition of $1 \mu \mathrm{l}$ of DRAQ5 (Biostatus Ltd.) for nuclear localization. Analyses were performed using an ImageStream 100 cytometer/fluorescence system (Amnis). In brief, debris and cell aggregates were excluded and individual cells were identified by gating on cell area and aspect ratio (ratio of length to width) as confirmed by brightfield (BF) microscopy according to the manufacturer's instructions. Analyses were performed following gating on DRAQ5 ${ }^{+}$and nonsaturated cells by flow cytometry. Individual cell populations were identified by gating on cells expressing given surface markers by flow cytometry and confirmed by visual inspection of the pattern of fluorescence on the cell surface. Area and N/C ratios for individual cells using computer-generated algorithms incorporating dimensions were determined using BF microscopy and DRAQ5 channels (Amnis). LANA positivity was defined by visual confirmation of characteristic punctate intranuclear staining of individual cells limited to areas of staining less than 7 pixels in the largest dimension in order to rigorously exclude nonspecific staining.

Statistics. Statistical comparisons for morphologic characteristics of cells were performed with GraphPad Prism software version 4.0a using the MannWhitney $U$ nonparametric $t$ test, assuming a non-Gaussian distribution of data, to calculate 2 -tailed $P$ values (significance defined as $P<0.05$ ).

Construction of NOD/SCID-bu mice. Six- to eight-week-old NOD/SCID (NOD/LtSz-Prkdcscid/J or NOD/SCID, Jackson Laboratory) were purchased and maintained in pathogen-free conditions. Trimethoprim-sulfamethoxazole $(3 \mathrm{mg} / \mathrm{ml})$ and neomycin $(2 \mathrm{mg} / \mathrm{ml})$ (Sigma-Aldrich) in sterile water were provided for routine consumption for a minimum of 2 weeks prior to surgery. On the day of surgery, mice received $325 \mathrm{cGy}$ of whole-body $\gamma$ irradiation (Gammacell-40; Nordion). Cadaveric human fetal bone, thymus, and skin grafts of 18- to 22-week gestation, received from an NIH-approved procurement facility (Advanced Bioscience Resources) in accordance with US regulations, were stored in cold 1640 RPMI. At the time of surgery, mice were anesthetized intraperitoneally with $16 \mu \mathrm{l} / \mathrm{g}$ of a $5 \%$ ketamine $/ 2.5 \%$ xylazine solution in sterile water and maintained under general anesthesia with inhaled methoxyflurane (Janssen). Human tissue grafts were processed and implanted subcutaneously in recipient animals as described elsewhere (29), and incision sites were closed with biodegradable cyanoacrylate (Vetbond; $3 \mathrm{M}$ ). The University of Virginia Animal Care and Use Committee approved all animal housing and surgical procedures in accordance with University of Virginia guidelines.

Identification of total and anti-LANA-specific buman IgG. Plasma samples were obtained from NOD/SCID-hu mice following centrifugation of whole blood and stored at $-20^{\circ} \mathrm{C}$. Samples were tested for human IgG using a commercially available ELISA according to the manufacturer's instructions (ZeptoMetrix Corp.). Colorimetric signals were quantified using an optical density plate reader at $260 \mathrm{~nm}$ in duplicate for each sample and concentrations determined based on standard curves using serially diluted purified human IgG. To screen for human KSHV-specific humoral responses, standard LANA IFAs were performed as we have described previously but with whole BCBL-1 cells rather than isolated nuclei. In brief, duplicate wells with fixed (in 1:1 methanol/acetone) BCBL-1 cells were incubated for 60 minutes with serial dilutions of plasma from KSHV- or PBS-injected mice or archived sera from human patients with KS and documented anti-LANA antibodies, as indicated in Figure 6. Additional wells were incubated with rabbit polyclonal antiLANA IgG antibodies (generously provided by Don Ganem, UCSF, San Francisco, California, USA). Secondary antibodies included goat antihuman, anti-mouse, or anti-rabbit IgG conjugated to Texas Red (Jackson ImmunoResearch Laboratories Inc.), and nuclei were stained with DAPI. Additional controls included KSHV-negative BJAB cells incubated with serum from KSHV-injected NOD/SCID-hu mice and Texas Red-conjugated goat anti-human IgG secondary antibodies. Slides were kept in the dark at $4^{\circ} \mathrm{C}$ for more than 48 hours before review, using the same deconvolution and compression methodology as above.

GCV administration to NOD/SCID-bu mice. For a subset of NOD/SCIDhu mice, $25 \mathrm{mg} / \mathrm{kg} \mathrm{GCV}$ (Cytovene-IV; Roche Diagnostics) was administered twice daily by intraperitoneal injection beginning 3 days before and extending 1 day beyond the 3 weekly KSHV injections as described above for unimplanted animals. 


\section{Acknowledgments}

This research received grant support from the NIH (5R-01CA88768 to D.H. Kedes and K08-1CA103858-01A1 to C.H. Parsons). L.A. Adang is an MSTP trainee (T32 GM 07267-27). The research received additional support from the University of Virginia Cancer Center (P30 CA44579). We would also like to thank members of the University of Virginia Research Histology Core Facility, Joanne Lannigan for her advice regarding flow cytometry experiments, Michael G. Brown for his advice regarding NK cell analyses, and William Newcomb for his help and advice with electron microscopy.
Received for publication October 28, 2005, and accepted in revised form May 9, 2006.

Address correspondence to: Dean H. Kedes, University of Virginia, 1300 Jefferson Park Avenue, Box 800734, Jordan Hall, Room 7069, Charlottesville, Virginia 22908, USA. Phone: (434) 243-2758; Fax: (434) 982-1071; E-mail: kedes@virginia.edu.

J. Robert Taylor Jr.'s present address is: Virology-BioProduction, American Type Culture Collection, Manassas, Virginia, USA.
1. Okano, M., and Gross, T.G. 2000. A review of Epstein-Barr virus infection in patients with immunodeficiency disorders. Am. J. Med. Sci. 319:392-396.

2. Boshoff, C., and Chang, Y. 2001. Kaposi's sarcomaassociated herpesvirus: a new DNA tumor virus. Annu. Rev. Med. 52:453-470.

3. Gandhi, M.K., and Khanna, R. 2004. Human cytomegalovirus: clinical aspects, immune regulation, and emerging treatments. Lancet Infect. Dis. 4:725-738.

4. Cesarman, E., Chang, Y., Moore, P.S., Said, J.W., and Knowles, D.M. 1995. Kaposi's sarcoma-associated herpesvirus-like DNA sequences in AIDS-related body-cavity-based lymphomas. N. Engl. J. Med. 332:1186-1191.

5. Cesarman, E., and Knowles, D.M. 1999. The role of Kaposi's sarcoma-associated herpesvirus (KSHV/ HHV-8) in lymphoproliferative diseases. Semin. Cancer Biol. 9:165-174.

6. Chang, Y., et al. 1994. Identification of herpesviruslike DNA sequences in AIDS-associated Kaposi's sarcoma. Science. 266:1865-1869.

7. Cattelan, A.M., et al. 2005. Long-term clinical outcome of AIDS-related Kaposi's sarcoma during highly active antiretroviral therapy. Int. J. Oncol. 27:779-785.

8. Boulanger, E., et al. 2005. Prognostic factors and outcome of human herpesvirus 8-associated primary effusion lymphoma in patients with AIDS. J. Clin. Oncol. 23:4372-4380.

9. Chronowski, G.M., et al. 2001. Treatment of unicentric and multicentric Castleman disease and the role of radiotherapy. Cancer. 92:670-676.

10. Dittmer, D., et al. 1999. Experimental transmission of Kaposi's sarcoma-associated herpesvirus (KSHV/HHV-8) to SCID-hu Thy/Liv mice. J. Exp. Med. 190:1857-1868.

11. Picchio, G.R., et al. 1997. The KSHV/HHV8infected BCBL-1 lymphoma line causes tumors in SCID mice but fails to transmit virus to a human peripheral blood mononuclear cell graft. Virology. 238:22-29.

12. Renne, R., et al. 2004. Experimental transmission of Kaposi's sarcoma-associated herpesvirus (KSHV/HHV-8) to SIV-positive and SIV-negative rhesus macaques. J. Med. Primatol. 33:1-9.

13. Kedes, D.H., Lagunoff, M., Renne, R., and Ganem, D. 1997. Identification of the gene encoding the major latency-associated nuclear antigen of the Kaposi's sarcoma-associated herpesvirus. J. Clin. Invest. 100:2606-2610.

14. Lukac, D.M., Kirshner, J.R., and Ganem, D. 1999. Transcriptional activation by the product of open reading frame 50 of Kaposi's sarcoma-associated herpesvirus is required for lytic viral reactivation in B cells. J. Virol. 73:9348-9361.

15. Lo, P., Yu, X., Atanasov, I., Chandran, B., and Zhou, Z.H. 2003. Three-dimensional localization of pORF65 in Kaposi's sarcoma-associated herpesvirus capsid. J. Virol. 77:4291-4297.

16. Nealon, K., et al. 2001. Lytic replication of Kaposi's sarcoma-associated herpesvirus results in the formation of multiple capsid species: isolation and molecular characterization of A, B, and C capsids from a gammaherpesvirus. J. Virol. 75:2866-2878.

17. Trus, B.L., et al. 2001. Capsid structure of Kaposi's sarcoma-associated herpesvirus, a gammaherpesvirus, compared to those of an alphaherpesvirus, herpes simplex virus type 1 , and a betaherpesvirus, cytomegalovirus. J. Virol. 75:2879-2890.

18. Asahi-Ozaki, Y., Sato, Y., Kanno, T., Sata, T., and Katano, H. 2006. Quantitative analysis of Kaposi sarcoma-associated herpesvirus (KSHV) in KSHVassociated diseases. J. Infect. Dis. 193:773-782.

19. Wu, L., Renne, R., Ganem, D., and Forghani, B. 2000. Human herpesvirus 8 glycoprotein K8.1: expression, post-translational modification and localization analyzed by monoclonal antibody. J. Clin. Virol. 17:127-136.

20. Flano, E., Husain, S.M., Sample, J.T., Woodland, D.L., and Blackman, M.A. 2000. Latent murine gamma-herpesvirus infection is established in activated B cells, dendritic cells, and macrophages. J. Immunol. 165:1074-1081.

21. Flano, E., Kim, I.J., Moore, J., Woodland, D.L., and Blackman, M.A. 2003. Differential gamma-herpesvirus distribution in distinct anatomical locations and cell subsets during persistent infection in mice. J. Immunol. 170:3828-3834.

22. Monini, P., et al. 1999. Reactivation and persistence of human herpesvirus- 8 infection in B cells and monocytes by Th- 1 cytokines increased in Kaposi's sarcoma. Blood. 93:4044-4058.

23. Rappocciolo, G., et al. 2006. DC-SIGN is a receptor for human herpesvirus 8 on dendritic cells and macrophages. J. Immunol. 176:1741-1749.

24. Lin, Y., Chen, Y., Zeng, Y., Wang, T., and Zeng, S. 2005. Lymphocyte phenotyping and NK cell activity analysis in pregnant NOD/SCID mice. J. Reprod. Immunol. 68:39-51.

25. Sirianni, M.C., et al. 2002. NK cell activity controls human herpesvirus 8 latent infection and is restored upon highly active antiretroviral therapy in AIDS patients with regressing Kaposi's sarcoma. Eur. J. Immunol. 32:2711-2720.

26. Shultz, L.D., et al. 1995. Multiple defects in innate and adaptive immunologic function in NOD/LtSzscid mice. J. Immunol. 154:180-191.

27. Henry, M., et al. 1999. Infection of circulating CD34+ cells by HHV-8 in patients with Kaposi's sarcoma. J. Invest. Dermatol. 113:613-616.

28. Ye, Z.J., Kluger, Y., Lian, Z., and Weissman, S.M. 2005. Two types of precursor cells in a multipotential hematopoietic cell line. Proc. Natl. Acad. Sci. U. S. A. 102:18461-18466.

29. Roncarolo, M.G., and Carballido, J.M. 1998. Construction of human-SCID chimeric mice. In Current protocols in immunology. J.E. Coligan, A.M. Kruisbeek, D.H. Margulies, E.M. Shevach, and W. Strober, editors. John Wiley. New York, New York, USA. 4.8.1-4.8.17.

30. Kedes, D.H., et al. 1996. The seroepidemiology of human herpesvirus 8 (Kaposi's sarcoma-associated herpesvirus): distribution of infection in KS risk groups and evidence for sexual transmission. Nat. Med. 2:918-924.

31. Kulkarni, A.B., Holmes, K.L., Fredrickson, T.N.,
Hartley, J.W., and Morse, H.C., 3rd. 1997. Characteristics of a murine gammaherpesvirus infection immunocompromised mice. In Vivo. 11:281-291.

32. Nash, A.A., Dutia, B.M., Stewart, J.P., and Davison, A.J. 2001. Natural history of murine gamma-herpesvirus infection. Philos. Trans. R. Soc. Lond. B Biol. Sci. 356:569-579.

33. Oda, W., et al. 2005. Analysis of genomic homology of murine gammaherpesvirus (MHV)-72 to MHV-68 and impact of MHV-72 on the survival and tumorigenesis in the MHV-72-infected CB17 scid/scid and CB17+/+ mice. Pathol. Int. 55:558-568.

34. Verzijl, D., et al. 2004. Differential activation of murine herpesvirus 68- and Kaposi's sarcoma-associated herpesvirus-encoded ORF74 G protein-coupled receptors by human and murine chemokines. J. Virol. 78:3343-3351.

35. Wong, S.W., et al. 1999. Induction of B cell hyperplasia in simian immunodeficiency virus-infected rhesus macaques with the simian homologue of Kaposi's sarcoma-associated herpesvirus. J. Exp. Med. 190:827-840.

36. Chugh, P., et al. 2005. Constitutive NF-kappaB activation, normal Fas-induced apoptosis, and increased incidence of lymphoma in human herpes virus 8 K13 transgenic mice. Proc. Natl. Acad. Sci. U. S. A. 102:12885-12890.

37. Fakhari, F.D., Jeong, J.H., Kanan, Y., and Dittmer, D.P. 2006. The latency-associated nuclear antigen of Kaposi sarcoma-associated herpesvirus induces B cell hyperplasia and lymphoma. J. Clin. Invest. 116:735-742. doi:10.1172/JCI26190.

38. Prakash, O., et al. 2005. Activation of Src kinase Lyn by the Kaposi sarcoma-associated herpesvirus K1 protein: implications for lymphomagenesis. Blood. 105:3987-3994.

39. Boshoff, C., et al. 1998. Establishing a KSHV+ cell line (BCP-1) from peripheral blood and characterizing its growth in Nod/SCID mice. Blood. 91:1671-1679.

40. D’Agostino, G., et al. 1999. Type I consensus IFN (IFN-con1) gene transfer into KSHV/HHV-8-infected BCBL-1 cells causes inhibition of viral lytic cycle activation via induction of apoptosis and abrogates tumorigenicity in SCID mice. J. Interferon Cytokine Res. 19:1305-1316.

41. Staudt, M.R., et al. 2004. The tumor microenvironment controls primary effusion lymphoma growth in vivo. Cancer Res. 64:4790-4799.

42. Foreman, K.E., et al. 2001. Injection of human herpesvirus-8 in human skin engrafted on SCID mice induces Kaposi's sarcoma-like lesions. J. Dermatol. Sci. 26:182-193.

43. Krithivas, A., Fujimuro, M., Weidner, M., Young, D.B., and Hayward, S.D. 2002. Protein interactions targeting the latency-associated nuclear antigen of Kaposi's sarcoma-associated herpesvirus to cell chromosomes. J. Virol. 76:11596-11604.

44. Dittmer, D.P. 2003. Transcription profile of Kaposi's sarcoma-associated herpesvirus in primary Kaposi's sarcoma lesions as determined by realtime PCR arrays. Cancer Res. 63:2010-2015.

45. Sun, R., et al. 1999. Kinetics of Kaposi's sarcomaassociated herpesvirus gene expression. J. Virol. 73:2232-2242. 
46. Zhong, W., Wang, H., Herndier, B., and Ganem, D. 1996. Restricted expression of Kaposi sarcomaassociated herpesvirus (human herpesvirus 8) genes in Kaposi sarcoma. Proc. Natl. Acad. Sci. U. S. A. 93:6641-6646.

47. Orenstein, J.M., et al. 1997. Visualization of human herpesvirus type 8 in Kaposi's sarcoma by light and transmission electron microscopy. AIDS. 11:F35-F45.

48. Engels, E.A., et al. 2003. Detection and quantification of Kaposi's sarcoma-associated herpesvirus to predict AIDS-associated Kaposi's sarcoma. AIDS. 17:1847-1851.

49. Stebbing, J., et al. 2003. Natural killer cells are not infected by Kaposi's sarcoma-associated herpesvirus in vivo, and natural killer cell counts do not correlate with the risk of developing Kaposi's sarcoma. AIDS. 17:1998-2000.

50. Akula, S.M., Pramod, N.P., Wang, F.Z., and Chandran, B. 2002. Integrin alpha3beta 1 (CD 49c/29) is a cellular receptor for Kaposi's sarcoma-associated herpesvirus (KSHV/HHV-8) entry into the target cells. Cell. 108:407-419.

51. Birkmann, A., et al. 2001. Cell surface heparan sulfate is a receptor for human herpesvirus 8 and interacts with envelope glycoprotein K8.1. J. Virol. 75:11583-11593.

52. Carballido, J.M., et al. 2000. Generation of primary antigen-specific human $\mathrm{T}$ - and $\mathrm{B}$-cell responses in immunocompetent SCID-hu mice. Nat. Med. 6:103-106.

53. Renwick, N., et al. 1998. Seroconversion for human herpesvirus 8 during HIV infection is highly predictive of Kaposi's sarcoma. AIDS. 12:2481-2488.

54. Kedes, D.H., and Ganem, D. 1997. Sensitivity of Kaposi's sarcoma-associated herpesvirus replication to antiviral drugs. Implications for potential therapy. J. Clin. Invest. 99:2082-2086.

55. Grundhoff, A., and Ganem, D. 2004. Inefficient establishment of KSHV latency suggests an additional role for continued lytic replication in Kaposi sarcoma pathogenesis. J. Clin. Invest. 113:124-136. doi:10.1172/JCI200417803.

56. Mocroft, A., et al. 1996. Anti-herpesvirus treatment and risk of Kaposi's sarcoma in HIV infection. Royal Free/Chelsea and Westminster Hospitals Collaborative Group. AIDS. 10:1101-1105.

57. Renne, R., et al. 1996. Lytic growth of Kaposi's sarcoma-associated herpesvirus (human herpesvirus 8 ) in culture. Nat. Med. 2:342-346.

58. Tomescu, C., Law, W.K., and Kedes, D.H. 2003. Surface downregulation of major histocompatibility complex class I, PE-CAM, and ICAM-1 following de novo infection of endothelial cells with Kaposi's sarcoma-associated herpesvirus. J. Virol. 77:9669-9684

59. O'Connor, C.M., Damania, B., and Kedes, D.H. 2003. De novo infection with rhesus monkey rhadinovirus leads to the accumulation of multiple intranuclear capsid species during lytic replication but favors the release of genome-containing virions. J. Virol. 77:13439-13447. 